\title{
Erratum to: The Use of F-18 Choline PET in the Assessment of Bone Metastases in Prostate Cancer: Correlation with Morphological Changes on CT
}

Mohsen Beheshti, ${ }^{1}$ Reza Vali, ${ }^{1}$ Peter Waldenberger, ${ }^{2}$ Friedrich Fitz, ${ }^{1}$ Michael Nader, ${ }^{1}$ Josef Hammer, ${ }^{3}$ Wolfgang Loidl, ${ }^{4}$ Christian Pirich, ${ }^{5}$ Ignac Fogelman, ${ }^{6}$

Werner Langsteger ${ }^{1}$

${ }^{1}$ Nuclear Medicine \& Endocrinology, PET/CT Center Linz, St. Vincent's Hospital, Linz, Austria

${ }^{2}$ Radiology, St. Vincent's Hospital, Linz, Austria

${ }^{3}$ Radiation Oncology, St. Vincent's Hospital, Linz, Austria

${ }^{4}$ Urology, St. Vincent's Hospital, Linz, Austria

${ }^{5}$ Nuclear Medicine \& Endocrinology, Paracelsus Private Medical University, Salzburg, Austria

${ }^{6}$ Division of Imaging, King's College, London, UK

$\mathrm{D}$ ue to an error in production, this article was published twice: first as DOI 10.1007/s11307-009-0217-0 [Mol Imaging Biol (2009) 11:446-454] and then as DOI 10.1007/ s11307-009-0239-7 [Mol Imaging Biol (2010) 12:98-107].

DOI 10.1007/s11307-009-0217-0 should be considered the article of record.

The Editor regrets this error. 